PROCEEDINGS OF THE

AMERICAN MATHEMATICAL SOCIETY

Volume 134, Number 12, December 2006, Pages 3715-3719

S 0002-9939(06)08596-0

Article electronically published on June 15, 2006

\title{
A COUNTEREXAMPLE RELATED TO TOPOLOGICAL SUMS
}

\author{
SHUJI YAMAMOTO AND ATSUSHI YAMASHITA
}

(Communicated by Alexander Dranishnikov)

\begin{abstract}
In this paper we construct compact metric spaces $X, Y$ which are topologically distinct but whose topological sums $X \sqcup X$ and $Y \sqcup Y$ are homeomorphic.
\end{abstract}

\section{IntRoduction AND the MAIN RESUlt}

For topological spaces $X, Y$, we denote by $X \sqcup Y$ the topological sum of $X$ and $Y$. Let $X$ and $Y$ be topological spaces and assume that $X \sqcup X$ and $Y \sqcup Y$ are homeomorphic (we denote this by $X \sqcup X \approx Y \sqcup Y$ ). Then, one may ask whether $X \approx Y$ holds.

If $X$ is a connected space, obviously, this is always true. It seems that even for "most" disconnected spaces $X, Y$ such that $X \sqcup X \approx Y \sqcup Y, X$ and $Y$ are homeomorphic. But this is not true in general; we will describe a counterexample.

Theorem 1.1. There exist compact metric spaces $X, Y$ which satisfy the following property: $X$ and $Y$ are nonhomeomorphic, but the topological sums $X \sqcup X$ and $Y \sqcup Y$ are homeomorphic. Moreover, such spaces $X, Y$ can be constructed as subspaces of the Euclidean plane $\mathbb{R}^{2}$.

In this paper, for notational convenience, we will construct an example of the pair $X, Y$ as subspaces of $\mathbb{R}^{4}$. However, it will be easily observed that one can construct $X, Y$ with the same property as (compact) subspaces of $\mathbb{R}^{2}$ in a similar way.

For $n \geqq 0$, we denote by

$$
\bigsqcup_{n} X
$$

the topological sum of $n$ copies of $X$. If $n=0$, this means the empty set $\emptyset$. Let $\mathbb{N}, \mathbb{Z}, \mathbb{Q}$, and $\mathbb{R}$ be the set of all positive integers, all integers, all rational numbers and all real numbers, respectively. Let $I=[0,1]=\{x \in \mathbb{R} ; 0 \leqq x \leqq 1\}$ be the closed interval between 0 and 1 . For points $x, y$ in the Euclidean space $\mathbb{R}^{n}$, put

$$
\langle x, y\rangle=\{(1-t) x+t y ; t \in I\} \subset \mathbb{R}^{n} .
$$

Received by the editors September 27, 2004 and, in revised form, July 9, 2005.

2000 Mathematics Subject Classification. Primary 54B99, 54G15, 54G20.

Key words and phrases. Topological sum, disjoint union, counterexample.

The authors are pleased to thank the referee and K. Sakai for their useful comments. The authors also thank S. Saito, who corrected English expressions and many errors in the preliminary version of this paper.

(C)2006 American Mathematical Society Reverts to public domain 28 years from publication 


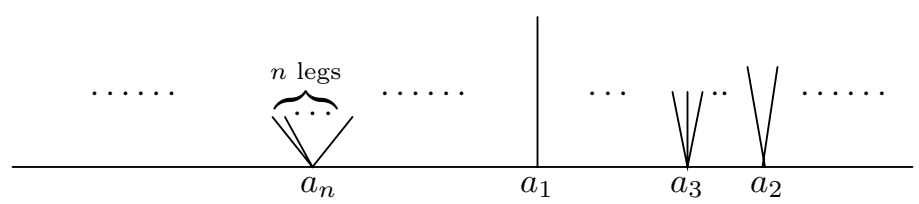

Figure 1 . The space $Z$

\section{Construction of the example}

The main idea is to construct spaces $X$ and $Z$ which have the following property:

Property A. The spaces $X$ and $X \sqcup Z$ are nonhomeomorphic, but $X$ and $X \sqcup Z \sqcup Z$ are homeomorphic.

If we can construct such a pair of spaces $X$ and $Z$, the spaces $X$ and $Y=X \sqcup Z$ clearly form a counterexample. Now, the problem is to construct $X$ and $Z$ satisfying Property A.

The space $Z$ is constructed as follows. Arrange the countable set $I \cap \mathbb{Q}$ to get a sequence $a_{1}, a_{2}, a_{3}, \ldots$ Then let $Z$ be the closed interval $I$ with $n$ "legs" at each rational point $a_{n}$ (see Figure 1). Precisely, let $v_{i}=(1,1 / i) \in \mathbb{R}^{2}$ for $i \in \mathbb{N}$ and let

$$
F_{n}=\bigcup_{i=1}^{n}\left\langle 0, \frac{1}{n} v_{i}\right\rangle \subset \mathbb{R}^{2}
$$

for $n \in \mathbb{N}$. Then we put

$$
Z=(I \times\{(0,0)\}) \cup \bigcup_{n \in \mathbb{N}}\left(\left\{a_{n}\right\} \times F_{n}\right) \subset \mathbb{R}^{3} .
$$

Observe that every open cover of $Z$ has a finite subcover, whence $Z$ is compact.

The following lemma is easily seen by counting the path components of the complements of each point of $Z$ (especially, the "node" $\left(a_{n}, 0,0\right)$ of $Z$ for each $n \in \mathbb{N}$ ) and observing that the set $\left\{a_{n} ; n \in \mathbb{N}\right\}=I \cap \mathbb{Q}$ is dense in $I$.

Lemma 2.1. Every homeomorphism $\varphi: Z \longrightarrow Z$ fixes the points of $I \times\{(0,0)\}$.

The other space $X$ has countably many copies of $Z$ as subspaces. Precisely, we construct $X$ as follows. Let $f: I \longrightarrow I$ be the homeomorphism defined by

$$
f(x)= \begin{cases}x / 2 & \text { if } 0 \leqq x \leqq 1 / 2 \\ (3 x-1) / 2 & \text { if } 1 / 2 \leqq x \leqq 1\end{cases}
$$

(See Figure 2.) Clearly, $f$ induces a homeomorphism from $I \cap \mathbb{Q}$ to itself. Let $f^{0}=\operatorname{id}_{I}$, the identity map of $I, f^{1}=f$ and $f^{n}=f \circ f^{n-1}$ for integers $n \geqq 2$. Define $f^{-n}=\left(f^{n}\right)^{-1}$ for each $n \in \mathbb{N}$. Each $f^{n}$ is a homeomorphism from $I$ to itself and induces a homeomorphism from $I \cap \mathbb{Q}$ to itself.

Let

$$
X_{m}=(I \times\{(0,0)\}) \cup \bigcup_{n \in \mathbb{N}}\left(\left\{f^{m-1}\left(a_{n}\right)\right\} \times \frac{1}{m} F_{n}\right) \subset \mathbb{R}^{3}
$$

for each $m \in \mathbb{N}$ and let

$$
X=(\{0\} \times I \times\{(0,0)\}) \cup \bigcup_{m \in \mathbb{Z} \backslash\{0\}}\left(\left\{\frac{1}{m}\right\} \times X_{|m|}\right) \subset \mathbb{R}^{4} .
$$




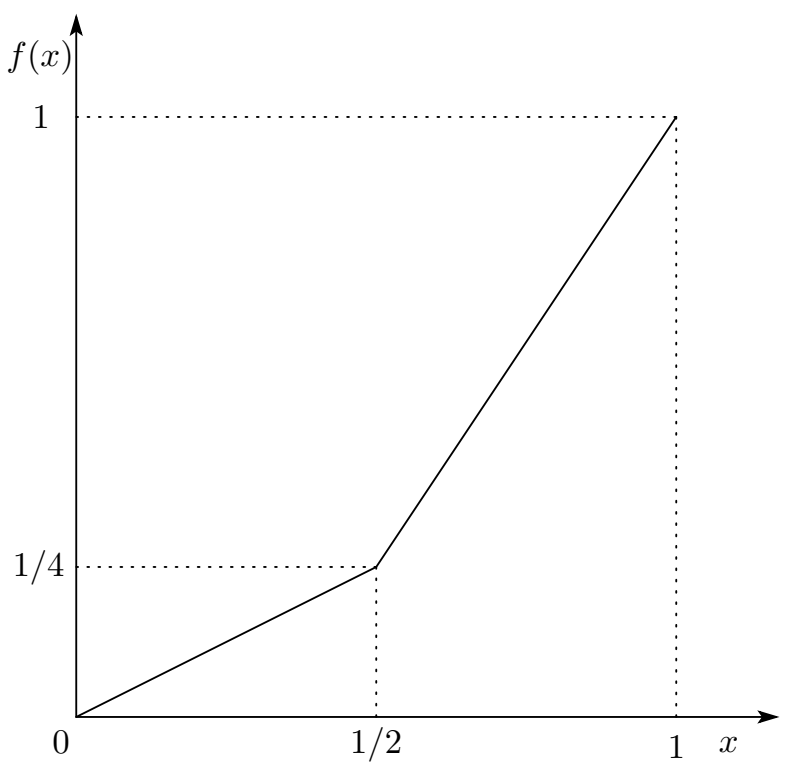

Figure 2. The homeomorphism $f$

Then, clearly, $I_{0}=\{0\} \times I \times\{(0,0)\}$ and

$$
Z_{m}=\left\{\frac{1}{m}\right\} \times X_{|m|} \quad(m \in \mathbb{Z} \backslash\{0\})
$$

are path components of $X$. We easily see that $X$ is closed in $\mathbb{R}^{4}$. Since $X$ is a bounded subset of $\mathbb{R}^{4}, X$ is compact (see Figure 3 ).

Note that the map $Z \longrightarrow Z_{m}$ given by

$$
(x, y, z) \longmapsto\left(1 / m, f^{|m|-1}(x), y /|m|, z /|m|\right)
$$

is a homeomorphism for each $m \in \mathbb{Z} \backslash\{0\}$. By this fact and Lemma 2.1, we have

Lemma 2.2. If $m, m^{\prime} \in \mathbb{Z} \backslash\{0\}$, every homeomorphism $Z_{m} \longrightarrow Z_{m^{\prime}}$ induces the homeomorphism $\{1 / m\} \times I \times\{(0,0)\} \longrightarrow\left\{1 / m^{\prime}\right\} \times I \times\{(0,0)\}$ given by

$$
\left(\frac{1}{m}, x, 0,0\right) \longmapsto\left(\frac{1}{m^{\prime}}, f^{\left|m^{\prime}\right|-|m|}(x), 0,0\right) .
$$

Observe that $\ldots, f^{-2}(x), f^{-1}(x), x, f^{1}(x), f^{2}(x), \ldots$ are distinct in $I$ for each $x \in I \backslash\{0,1\}$. The next proposition follows from this fact and Lemma 2.2

Proposition 2.3. Assume that $m, m^{\prime} \in \mathbb{Z} \backslash\{0\}$ and that $\psi: Z_{m} \longrightarrow Z_{m^{\prime}}$ is a homeomorphism. If there exists a point $x \in I \backslash\{0,1\}$ such that

$$
\psi\left(\frac{1}{m}, x, 0,0\right)=\left(\frac{1}{m^{\prime}}, f^{n}(x), 0,0\right)
$$

then $\left|m^{\prime}\right|-|m|=n$.

Now, we can prove the following essential proposition.

Proposition 2.4. The space $X \sqcup \bigsqcup_{N} Z$ is homeomorphic to $X$ if and only if $N$ is even. In particular, $X$ and $Z$ satisfy Property $A$. 

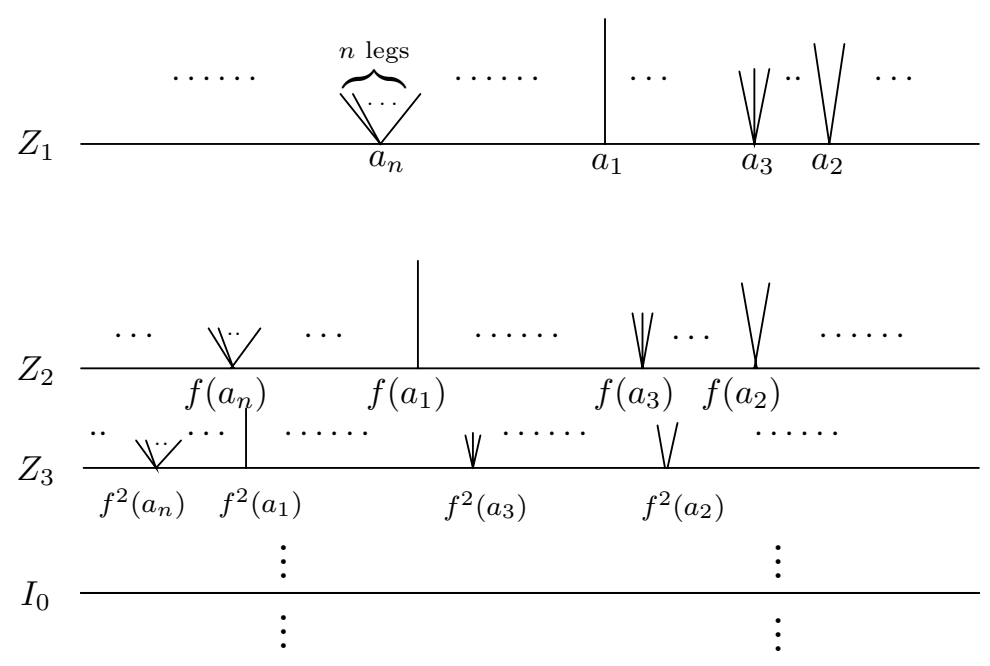

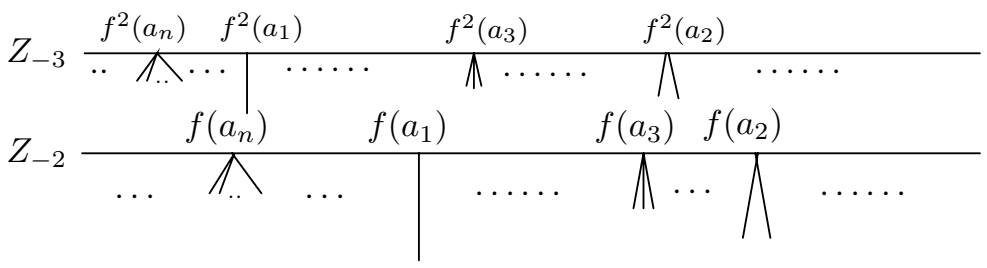

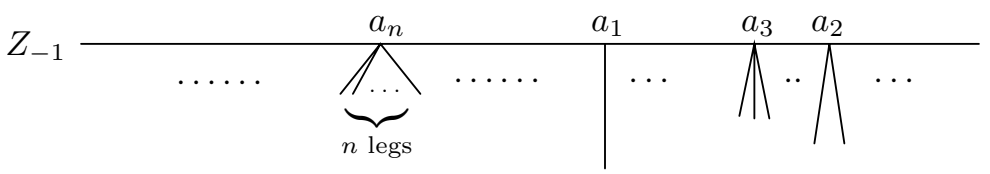

Figure 3. The space $X$

Proof. First, assume that $N=2 n$ is an even positive integer. Define a map $\varphi: X \longrightarrow X$ by $\varphi(0, x, 0,0)=\left(0, f^{n}(x), 0,0\right)$ for $x \in I$ and

$$
\varphi\left(\frac{1}{m}, x, y, z\right)= \begin{cases}\left(\frac{1}{m+n}, f^{n}(x), \frac{m}{m+n} y, \frac{m}{m+n} z\right) & \text { if } m>0, \\ \left.\frac{1}{m-n}, f^{n}(x), \frac{m}{m-n} y, \frac{m}{m-n} z\right) & \text { if } m<0 .\end{cases}
$$

This $\varphi$ is clearly injective and satisfies

$$
\varphi(X)=X \backslash \bigcup_{0<|m|<n} Z_{m} .
$$

Clearly, in order to prove the "if" part, it suffices to show that $\varphi$ is a homeomorphism onto its image. This is trivial because $\varphi$ is continuous and $X$ is compact.

To see the "only if" part, suppose that $\bigsqcup_{N} Z \sqcup X \approx X$.

Let $\varphi$ be a homeomorphism

$$
\varphi: \bigsqcup_{N} Z \sqcup X \longrightarrow X .
$$


Since homeomorphisms take each path component onto another one and $I_{0} \approx I \not z$ $Z$, each $Z_{m}$ is mapped onto some $Z_{m^{\prime}}$, and $I_{0}$ onto $I_{0}$ by $\varphi$. Take $x \in I$ so that $\varphi^{-1}(0,1 / 2,0,0)=(0, x, 0,0)$. Then $x \notin\{0,1\}$.

Define a sequence $\left\{p_{j}\right\}_{j \in \mathbb{N}}$ in $X$ as follows: $p_{2 k}=(1 / k, x, 0,0)$ and $p_{2 k-1}=$ $(-1 / k, x, 0,0)$. Obviously, $\left\{p_{j}\right\}$ converges to $(0, x, 0,0) \in I_{0}$. Because $\varphi$ is continuous, $\left\{\varphi\left(p_{j}\right)\right\}$ must converge to $\varphi(0, x, 0,0)=(0,1 / 2,0,0)$. Use Lemma 2.2 to observe that each $\operatorname{pr}_{2}\left(\varphi\left(p_{j}\right)\right)$ must belong to the set $A=\left\{f^{n}(x) ; n \in \mathbb{Z}\right\} \subset I$, where $\mathrm{pr}_{2}$ denotes the second projection $\mathbb{R}^{4} \longrightarrow \mathbb{R}$. Since the closure of $A$ in $I$ is $A \cup\{0,1\}$, the point $1 / 2$ belongs to $A \cup\{0,1\}$, which means that $1 / 2 \in A$. Hence, there is an integer $n$ such that $1 / 2=f^{n}(x)$. Since $1 / 2$ is an isolated point of $A$ and $\operatorname{pr}_{2}\left(\varphi\left(p_{j}\right)\right) \rightarrow 1 / 2$ as $j \rightarrow \infty, \operatorname{pr}_{2}\left(\varphi\left(p_{j}\right)\right)=1 / 2$ for $j \geqq 2 M-1$, where $M$ is a sufficiently large number.

Take any integer $m$ such that $|m| \geqq M$. Then there exists a number $j$ such that $p_{j} \in Z_{m}$. This $j$ satisfies $j \geqq 2 M-1$ and $\operatorname{pr}_{2}\left(\varphi\left(p_{j}\right)\right)=1 / 2$. Pick $m^{\prime} \in \mathbb{Z}$ so that $\varphi\left(p_{j}\right)$ belongs to $Z_{m^{\prime}}$. Then, $\varphi$ maps $Z_{m}$ onto $Z_{m^{\prime}}$ homeomorphically. Notice that $\operatorname{pr}_{2}\left(p_{j}\right)=x$ and $\operatorname{pr}_{2}\left(\varphi\left(p_{j}\right)\right)=1 / 2=f^{n}(x)$. By Proposition 2.3, we have $\left|m^{\prime}\right|-|m|=n$. This means that $\varphi\left(Z_{m} \cup Z_{-m}\right)=Z_{m+n} \cup Z_{-(m+n)}$ if $m \geqq M$.

Then, we have

$$
\varphi\left(\bigcup_{|m| \geqq M} Z_{m}\right)=\bigcup_{|m| \geqq M+n} Z_{m},
$$

and hence,

$$
\varphi\left(\bigsqcup_{N} Z \cup \bigcup_{0<|m|<M} Z_{m}\right)=\bigcup_{0<|m|<M+n} Z_{m} .
$$

In $(\sharp)$, since $\varphi$ is a homeomorphism, the numbers of path components within the bracket in the left-hand side and in the right-hand side must be the same. This means

$$
N+2 M-2=2 M+2 n-2,
$$

that is, $N=2 n$. Therefore, $N$ is even.

Letting $n=1$ or 2, we see from Proposition 2.4 that $X \sqcup Z \sqcup Z \approx X$, and that $X \sqcup Z \not \approx X$. This means that $X$ and $Z$ satisfy Property A. Hence, the spaces $X$ and $Y=X \sqcup Z$ give an example of the pair $X, Y$ in Theorem 1.1.

Finally we note that, by a slight modification, we have an example of compact metric spaces $X_{n}, Y_{n}(n \in \mathbb{N})$ such that $\bigsqcup_{k} X_{n} \not \bigsqcup_{k} Y_{n}$ for $k<n$ but $\bigsqcup_{n} X_{n} \approx$ $\bigsqcup_{n} Y_{n}$.

Graduate School of Mathematical Sciences, The University of Tokyo, 3-8-1, Komaba, MEguro-Ku, TOKYO 153-8914, JAPAN

E-mail address: yamashu@ms.u-tokyo.ac.jp

Graduate School of Mathematical Sciences, The University of Tokyo, 3-8-1, Komaba, MEGURO-KU, TOKYO 153-8914, JAPAN

E-mail address: yonster@ms.u-tokyo.ac.jp 